

\title{
Dimensionality reduction on ocean model's outputs: Application to motion estimation on satellite images
}

\author{
Isabelle Herlin, Etienne Huot
}

\section{To cite this version:}

Isabelle Herlin, Etienne Huot. Dimensionality reduction on ocean model's outputs: Application to motion estimation on satellite images. IGARSS - IEEE International Geoscience and Remote Sensing Symposium, Jul 2015, Milan, Italy. 10.1109/IGARSS.2015.7326469 hal-01174027

\section{HAL Id: hal-01174027 \\ https://hal.inria.fr/hal-01174027}

Submitted on 8 Jul 2015

HAL is a multi-disciplinary open access archive for the deposit and dissemination of scientific research documents, whether they are published or not. The documents may come from teaching and research institutions in France or abroad, or from public or private research centers.
L'archive ouverte pluridisciplinaire HAL, est destinée au dépôt et à la diffusion de documents scientifiques de niveau recherche, publiés ou non, émanant des établissements d'enseignement et de recherche français ou étrangers, des laboratoires publics ou privés. 


\title{
DIMENSIONALITY REDUCTION ON OCEAN MODEL'S OUTPUTS: APPLICATION TO MOTION ESTIMATION ON SATELLITE IMAGES
}

\author{
Isabelle Herlin $^{1} \quad$ Etienne Huot ${ }^{1,2}$ \\ ${ }^{1}$ INRIA, Institut National de Recherche en Informatique et Automatique, France \\ ${ }^{2}$ Université de Versailles - Saint-Quentin-en-Yvelines, France
}

\begin{abstract}
Motion fields describing the ocean surface dynamics live in vectorial spaces of high dimension. Consequently, their estimation from satellite images requires huge computational resources. The issue of dimensionality reduction, that is the determination of representative low dimensional structures in these high dimensional spaces, is of major importance for any application that demands real-time or short-term results. Proper Order Decomposition allows to determine such subspace of motion fields on which estimation may be assessed with reduced complexity. A reduced model is obtained by Galerkin projection of evolution equations on this subspace. Motion is estimated by assimilating the observed image sequence with the reduced model. The paper describes how to derive the reduced space from a database of ocean model's outputs and explains how to estimate surface circulation from satellite sequences. Results are given on images acquired on the Black Sea basin by NOAA-AVHRR sensors.
\end{abstract}

\section{INTRODUCTION}

Surface circulation of oceans has been assessed for long by experts. Seasonal processes are well known and global circulation maps are available, as the one displayed on Fig. 1 for the Black Sea basin. Deriving instantaneous surface circulation maps with high space resolution and computing shortterm forecasts is of major interest for a large range of applications, such as coastal monitoring and pollutant trajectory forecast. However, satisfying the space-time constraints required by operational applications prevents to make use of 3D models. Alternatives must be envisioned. First, 2D models may be applied for estimating the surface velocity from a



Fig. 1. Black Sea circulation. From Blokhina et al.[1]

satellite image sequence, as described for Black Sea by Korotaev et al. [2]. A large range of data assimilation methods that derive motion from image observations is shown in the literature [3, 4]. To further reduce complexity, Herlin et al. [5] make use of a sliding window method and of the Proper Order Decomposition, and compute the motion basis for a given subwindow from results on the previous one. The principal weakness of their method is that it requires availability of the full model, at pixel resolution, which is run on the first subwindow. A fixed basis, not depending on the studied sequence, may be considered for defining the reduced vectorial space of motion fields, as proposed in [6]. The size of the reduced space that is looked for is then related to the spatial scales of the studied processes. Surface circulation of Black Sea displaying mesoscale structures, a large size basis has to be considered for correctly describing local structures. This strong constraint decreases the reduced model's performance and its computation time becomes prohibitive for operational use. 
In this paper, we discuss the possibility to determine a small size reduced basis that is obtained by Proper Order Decomposition (POD) of a motion fields database. The database is constructed for characterizing accurately the surface circulation of the studied area, so that linear combinations of the basis elements obtained by POD accurately describe the motion function observed on satellite image sequences. Section 2 summarizes the method that has been defined to obtain the reduced motion basis. Section 3 describes the evolution equations that are used to describe satellite sequences, the model reduction on the POD basis and the data assimilation method that estimates motion from satellite images. Section 4 illustrates the results obtained when processing satellite images acquired by NOAA-AVHRR sensors on the Black Sea.

\section{REDUCED BASIS OF MOTION FIELDS}

Sea Level Anomaly (SLA) analysis maps were available from the MyOcean European project website ${ }^{1}$ and computed by assimilation of SLA measures with the MHI NASU Hydrodynamical model. Geostrophic motion (Coriolis forces are balanced by pressure gradient forces) is then computed from these maps in order to obtain a 1-year database of surface motion at 5 kilometer of resolution, that includes seasonal variations of surface circulation. Two SLA maps and the corresponding geostrophic motion fields are given on Fig. 2.


Fig. 2. Top: reanalysis of SLA. Bottom: geostrophic motion.

A POD is applied on the ensemble of motion fields included in the database. 100 modes are kept to determine the basis $\Phi$. That corresponds to $99 \%$ of the whole variance. Some basis elements are displayed on Fig. 3. The 100-dimensional

\footnotetext{
${ }^{1}$ http://www.myocean.eu/
}

vectorial space spanned by the orthonormal basis $\Phi$ is used as reduced space in order to represent motion fields.


Fig. 3. Streamline display of elements 1, 20, 60 and 80 of $\Phi$.

\section{DATA ASSIMILATION IN THE REDUCED MODEL}

Let consider the following assumptions on the dynamics displayed by image sequences: motion $\mathbf{w}(\mathbf{x}, t)$ is constant along pixels' trajectories and pixels' image values $I(\mathbf{x}, t)$ are transported by motion. This gives the two following partial differ- 
ential equations:

$$
\left\{\begin{array}{l}
\frac{\partial \mathbf{w}}{\partial t}(\mathbf{x}, t)+(\mathbf{w} \cdot \nabla) \mathbf{w}(\mathbf{x}, t)=0 \\
\frac{\partial I}{\partial t}(\mathbf{x}, t)+\mathbf{w} \cdot \nabla I(\mathbf{x}, t)=0
\end{array}\right.
$$

with $\nabla$ denoting the spatial gradient.

An orthonormal basis $\Psi$ is obtained from applying POD on $L$ image observations. The projection $\mathbf{w}^{p}$ of motion on the vectorial space spanned by $\Phi$ and the description of $I$ on the basis $\Psi$ are given by:

$$
\begin{aligned}
\mathbf{w}^{p}(\mathbf{x}, t) & =\sum_{i=1}^{100} a_{i}(t) \phi_{i}(\mathbf{x}) \\
I^{p}(\mathbf{x}, t) & =\sum_{j=1}^{L} b_{j}(t) \psi_{j}(\mathbf{x})
\end{aligned}
$$

After replacing in System (1) and defining $a(t)$ the vector of size $100\left(\begin{array}{lll}a_{1}(t) & \ldots & a_{100}(t)\end{array}\right)^{T}, b(t)$ the vector of size $L$ $\left(\begin{array}{lll}b_{1}(t) & \ldots & b_{L}(t)\end{array}\right)^{T}, B(k)$ the $100 \times 100$ matrix whose element $(i, j)$ is $B(k)_{i, j}=\left\langle\left(\phi_{i} \cdot \nabla\right) \phi_{j}, \phi_{k}\right\rangle$, and $G(l)$ the $100 \times$ $L$ matrix whose element $(i, j)$ is $G(l)_{i, j}=\left\langle\phi_{i} \cdot \nabla \psi_{j}, \psi_{l}\right\rangle$, we obtain:

$$
\begin{aligned}
& \left\{\begin{array}{l}
\frac{d a_{k}}{d t}(t)+a^{T} B(k) a=0, \quad k=1 \ldots 100 \\
\frac{d b_{l}}{d t}(t)+a^{T} G(l) b=0, \quad l=1 \ldots L
\end{array}\right. \\
& \quad \mathbf{X}_{R}(t)=\left(\begin{array}{l}
a(t) \\
b(t)
\end{array}\right) \text { is the reduced state vector that verifies: } \\
& \frac{d \mathbf{X}_{R}}{d t}(t)+\mathbb{M}_{R}\left(\mathbf{X}_{R}(t)\right)=0 \text { with } \mathbb{M}_{R} \text { the Galerkin projec- } \\
& \text { tion of Eqs. (1), on bases } \Phi \text { and } \Psi .
\end{aligned}
$$

Satellite acquisitions are described on the basis $\Psi$, according to the second equation of System (2) and the resulting vectors $b(t)$ are assimilated in $\mathbb{M}_{R}$ for estimating motion vectors $a(t)$ and retrieving motion fields $\mathbf{w}(t)$.

\section{RESULTS}

\subsection{Synthetic Experiment}

A sequence of synthetic snapshots, similar to satellite acquisitions, is obtained from a run of an image model [7]. One image of the sequence is given on Fig. 4(a). A region of interest is defined on the basin and depicted as a black rectangle on Fig. 5(a). It includes a vortex of mushroom shape and a surface current (see the white arrow near the Crimean coast on Fig 1). Ground truth on motion is available from the same run and displayed on Fig. 4(b) for the ROI. Motion is then estimated from the snapshots with our method and displayed on Fig. 4(c). Statistics on the discrepancy between estimation and ground truth confirm the validity of the approach: the average relative error on the velocity norm is less that $1 \%$ and the maximum of the angular error is less than 0.1 degree.



(a)



(b)

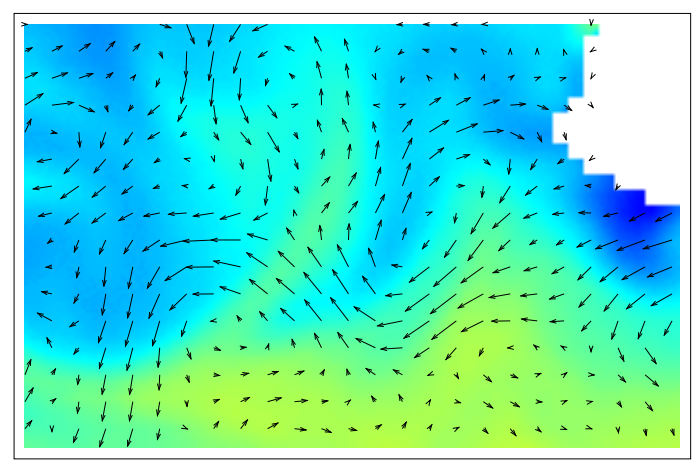

(c)

Fig. 4. (a): Synthetic image and ROI. (b): Ground truth. (c) Result of the reduced model. 


\subsection{Satellite Experiment}

The approach is then used for estimating motion on sequences of Sea Surface Temperature acquisitions. The demonstration is done on Fig. 5 with images acquired over Black Sea on July 14th and 15th 1998. Visual assessment of the results demonstrates the potential of the approach to detect and track the mesoscale structures on satellite data.



(a)



(b)



(c)

Fig. 5. Region of interest and estimated motion superposed on two consecutive images.

\section{CONCLUSION}

This paper describes motion estimation from satellite images and illustrates results on the whole Black Sea basin. The approach is based on a data assimilation method with a reduced model describing temporal evolution of velocity and image brightness. The reduced space for motion is obtained with a Proper Order Decomposition applied on a one-year motion database constructed from ocean model outputs. Images are described by their coefficients on a POD basis. The major perspectives concern the computation of seasonal spaces for representing motion in order to better assess the medium term variability and on the use of shallow water equations for describing surface circulation.

\section{REFERENCES}

[1] M. D. Blokhina and Y. D. Afanasyev, "Baroclinic instability and transient features of mesoscale surface circulation in the black sea: Laboratory experiment," Journal of Geophysical Research, vol. 108, no. C10, May 2003.

[2] G. Korotaev, E. Huot, F.-X. Le Dimet, I. Herlin, S. V. Stanichny, D. M. Solovyev, and L. Wu, "Retrieving ocean surface current by 4-D variational assimilation of sea surface temperature images," Remote Sensing of Environment (RSE), vol. 112, no. 4, pp. 1464-1475, April 2008, Special issue on data assimilation.

[3] O. Titaud, A. Vidard, I. Souopgui, and F.-X. Le Dimet, "Assimilation of image sequences in numerical models," Tellus A, vol. 62, pp. 30-47, 2010.

[4] N. Papadakis, T. Corpetti, and E. Mémin, "Dynamically consistent optical flow estimation," in International Conference on Computer Vision (ICCV), Rio de Janeiro, Brazil, Oct. 2007.

[5] I. Herlin and K. Drifi, "Learning reduced models for motion estimation on long temporal image sequences," in International Geoscience and Remote Sensing Symposium (IGARSS), Munich, Germany, July 2012.

[6] S. Kadri Harouna and V. Perrier, "Effective construction of divergence-free wavelets on the square," Journal of Computational and Applied Mathematics, vol. 240, pp. 74-86, March 2012.

[7] E. Huot, I. Herlin, and G. Papari, "Optimal orthogonal basis and image assimilation: Motion modeling," in International Conference on Computer Vision (ICCV), Sydney, Australia, December 2013. 